\title{
New Developments in the Treatment of Parkinson's Disease: Bridging the gap between Medical and Surgical Therapy for PD
}

\author{
Tsao-Wei Liang, MD; Ted Weissfeld, MD²; Timothy Bober ${ }^{3}$ \\ ${ }^{1}$ Department of Neurology, Thomas Jefferson University, Philadelphia, PA \\ ${ }^{2}$ Neurology Resident, Thomas Jefferson University, Philadelphia, PA \\ ${ }_{3}$ Medical Student, Sidney Kimmel Medical College at Thomas Jefferson University
}

\section{INTRODUCTION}

Parkinson's Disease (PD) is a progressive neurologic disease caused by loss of dopaminergic neurons in the midbrain substantia nigra. Decreased activation of dopamine dependent motor pathways results in the classic features of PD including tremor, bradykinesia, rigidity, and gait impairment. The gold standard treatment of Parkinson's Disease (PD) is levodopa, which acts to replenish deficient endogenous dopamine production, combined with carbidopa, a decarboxylase inhibitor to decrease peripheral breakdown of levodopa.

Early in the disease, the combination of carbidopa and levodopa (CD-LD) works remarkably well, with stable symptom control lasting longer than four hours. However, as the disease progresses, the response to levodopa begins to shorten and mirrors the half-life of CD-LD (roughly 90 minutes). This leads to extended "off" time without symptom control, and shorter periods of "on" time. ${ }^{1}$ Increasing frequency and/or doses of levodopa may be used to prolong "on" time, but this leads to increased dopaminergic side effects including dyskinesia. Dyskinesia and other motor complications including wearing-off occur in up to $90 \%$ of patients treated with levodopa for 5-10 years. ${ }^{1}$ Patients may ultimately experience rapid cycling between states of dyskinesia and "off" time, with minimal "on" time. ${ }^{2}$

Strategies to combat this rapid cycling center on extending the half-life and bioavailability of levodopa or using adjunctive medications to prolong dopaminergic activity, increasing trough levels while reducing peak blood levels that lead to dyskinesia. ${ }^{2}$ The original controlled release carbidopa-levodopa tablets (CR, ER, SA) lack the ability to provide immediate symptom that is often needed. ${ }^{3}$ Entacapone co-administration may prolong "on" time in advanced PD patients, ${ }^{4}$ but has been associated with an increased incidence of dyskinesia in early PD patients in the STRIDE-PD trial. ${ }^{5}$

A review of all adjunctive medications and their role in treating the fluctuations associated with PD is outside the scope of this article and we would refer readers to the American Academy of Neurology Practice Parameters and other reviews for further detail. ${ }^{6}$ An ideal therapy would provide a patient with continuous moderate dopaminergic activity, eliminating the symptoms and side effects associated with peaks and troughs. Continuous levodopa treatments have been shown to be superior to intermittent dosing for dyskinesias with Stocchi noting that an oral continuous method would be ideal. ${ }^{7}$

Deep brain stimulation (DBS) revolutionized PD treatment, providing thousands of patients with continuous motor benefits with less dyskinesia, with long-term studies showing sustained benefits. ${ }^{8}$ Nevertheless, a number of patients are not eligible for the procedure due to medical, neuropsychological, or even psychosocial contraindications. Please refer to the numerous guidelines currently available describing assessment of surgical candidacy. ${ }^{9,10}$ Additionally, eligible candidates may simply not be ready or interested in undergoing an invasive brain surgery. Fortunately in January 2015, two novel therapies (RYTARY and DUOPA) were introduced simultaneously in the United States that promise to bridge the gap between medical treatment and DBS therapy for PD.

\section{CARBIDOPA/LEVODOPA EXTENDED RELEASE CAPSULE}

IPX066, known by the trade name RYTARY, is a carbidopa-levodopa capsule manufactured by Impax Laboratories that is designed to provide a more sustained, less variable therapeutic level of levodopa. ${ }^{11}$ The mechanism of action is identical to other formulations of CD-LD as described above, however it is prepared as a multiparticulate capsule with both immediate and extended release components. ${ }^{12,13}$ It was developed to provide a longer duration of "on" time, less dyskinesia, and less need for adjunctive medications compared to standard CD-LD preparations. ${ }^{11}$

Early trials focused on the safety and pharmacokinetics of RYTARY. The initial open-label crossover comparison of RYTARY versus standard CD-LD formulations focused on safety, side effects, and pharmacokinetics. ${ }^{12,14}$ While limited by small sample size and open-label format, the results were very promising regarding the potential for RYTARY in providing sustained benefit to patients. Both RYTARY and immediate release CD-LD reached $50 \%$ peak LD plasma concentrations (Cmax) around 45 minutes; however, the duration of time spent above Cmax for RYTARY was 4 hours compared with 1.4 hours for immediate release CD-LD. When measured clinically, RYTARY provided a significant decrease in the Unified Parkinson's Disease Rating Scale (UPDRS) score 3-6 hours after a single dose compared to immediate release CD-LD, with more than half of patients still experiencing "on" time at 6 hours. ${ }^{14}$

This led to several randomized phase III trials of RYTARY in different clinical settings. ADVANCE-PD was a large (471 patients) multicenter, randomized, double-blind trial comparing RYTARY to immediate release CD-LD in PD patients with motor fluctuations. ${ }^{15}$ After open label dose titration to optimum doses of both RYTARY and immediate release CD-LD, 
patients were randomized to one treatment for 13 weeks. RYTARY provided a decrease in reported "off" time of 1.17 hours, an increase in "on" time without troublesome dyskinesia of 0.93 hours, and a decrease in UPDRS scale of 4.04 . The total Levodopa dose for RYTARY was $1630 \mathrm{mg}$ divided over 3.6 doses a day compared to $814.5 \mathrm{mg}$ over 5 doses for immediate release CD-LD. Side effect profiles and frequencies were similar between the groups. The major limitation in the study was the large dropout rate during transition from immediate release CD-LD to RYTARY, with $13 \%$ of patients unable to transition due to either side effects or poor efficacy.

APEX-PD was a large (381 patients) multicenter, randomized, double-blind trial assessing RYTARY as the initial dopaminergic therapy in early levodopa-naïve PD patients. ${ }^{13}$ Eligible patients with early stage PD (Hoehn and Yahr stage I, II, or III) who had not received any levodopa for 30 days were randomized to different doses of RYTARY or placebo. UPDRS parts II and III scores improved dramatically in the treatment arms versus placebo (between 11.7 and 14.9 for different doses) in addition to significantly improved quality of life scores. Higher doses of RYTARY were associated with increased side effects, however, which again resulted in increased dropout rates up to $15.3 \%$.

Finally, the ASCEND-PD trial by Stocchi et al. studied RYTARY in advanced PD patients who continued to have motor fluctuations despite stable regimens of CD-LD with entacapone. ${ }^{16}$ In this moderately sized (110 patients) randomized, double-blind, double dummy crossover study, patients spent two weeks receiving either RYTARY or CD-LD plus entacapone then switched treatments following a washout period. RYTARY was associated with a 1.4-hour decrease in "off" time and a 1.4-hour increase in "on" time without troublesome dyskinesia. Subjects on RYTARY averaged 3 doses a day totaling $1495 \mathrm{mg}$ levodopa compared with subjects on CD-LD plus entacapone who averaged 4-to-5 doses a day totaling 600-800 mg. Side effects were more prevalent in the RYTARY groups, with $20.2 \%$ of patients experiencing any side effect compared to $13.6 \%$ of CD-LD plus entacapone patients.
In all of these trials, side effect profiles were similar between RYTARY and other levodopa formulations. This is not unexpected, as the major side effects of nausea, vomiting, diarrhea, dizziness, falls, dyskinesia, confusion, and insomnia are likely secondary to dopaminergic activity. ${ }^{11,13,14,15,16,17}$ Slightly increased side effect frequency in the RYTARY arms may be due to higher total doses of levodopa, rapid dose titration, or the need for post-synaptic adjustments to the change in pharmacokinetics. Patients who completed the above trials were eligible to enroll in a nine-month open label extension by Waters et al., which confirmed the overall tolerability and safety of RYTARY over a longer treatment duration. ${ }^{18}$

Despite improved efficacy, similar side effect profiles, and reduced dose frequency, a major deterrent to initiating RYTARY is the transition period from immediate release CD-LD. The 10-15\% dropout rates seen with RYTARY in the above trials suggest that the transition to RYTARY may be difficult for some patients due to either poor initial efficacy during titration or side effects. However, the vast majority of patients were able to make the switch, with $87.2 \%$ successful from immediate release CD-LD and $82.7 \%$ from CD-LD entacapone formulations. ${ }^{19}$

Conversion from CD-LD to RYTARY should be based on a patient's total daily dose of immediate release levodopa. Slightly less than a two-to-one ratio of levodopa in RYTARY to immediaterelease CD-LD was used in the Hauser et al. trials, ${ }^{11,14,15}$ and is the recommended dosing conversion in the FDA prescribing information. ${ }^{20}$ In addition, patients often need further increases in doses after the initial switchover. To improve compliance in particularly sensitive patients, Hauser suggests changing only the AM dose from immediate release CD-LD to RYTARY and titrating that dose to desired effect. ${ }^{21}$ Once the optimal dose is found, the entire regimen can be changed to RYTARY without spending the entire day outside their therapeutic window. Patients starting RYTARY as their initial medication should take $95 \mathrm{mg}$ TID and increase to $145 \mathrm{mg}$ TID after 3 days if additional levodopa is needed. ${ }^{21}$ Like immediate-release CD-LD, RYTARY absorption is improved on an empty stomach due to less competition with protein. Alternatively, taking any levodopa preparation with food can alleviate $\mathrm{Gl}$ side effects if they occur.

\section{LEVODOPA-CARBIDOPA INTESTINAL GEL}

Levodopa-carbidopa intestinal gel (LCIG), is a novel CD-LD enteral suspension approved for the treatment of motor fluctuations in advanced Parkinson's disease (PD). Manufactured by AbbVie, Inc. in the U.S. as DUOPA, it has been available in select countries in the European Union since 2004 as DUODOPA. Powered by a CADD-1400 Legacy Pump, DUOPA allows for continuous 16-hour CD-LD administration into the proximal jejunum via a gastrostomy or PEG with a jejunal extension. The medication cassettes must be refrigerated prior to use and administered at room temperature. Standard daily dosing is divided into a morning dose, a daily 16-hour continuous dose, and additional boluses in case of inadequate response - each of which are calculated from the patient's standard daily dose of intermediate-release CD-LD and titrated to suit their needs. ${ }^{22}$

The efficacy of DUOPA in managing motor complications has been demonstrated in several studies. Olanow et. al. completed a 12-week, randomized, double-blinded, double dummy trial comparing DUOPA intestinal infusion to immediate-release CD-LD in 71 patients with advanced PD and greater than three hours of reported "off" time per day. ${ }^{23}$ After undergoing PEG-J placement, patients were randomized to receive daily 16-hour DUOPA infusion or immediaterelease oral CD-LD capsules with a matching placebo gel. Patients recorded their motor status every 30 minutes to indicate their status as "off," "on" without dyskinesia, "on" with troublesome dyskinesia, or sleeping. The LCIG group was found to have clinically significant reductions in "off" time from baseline and when compared to the controls $(-4.04$ hours from baseline, -1.91 hours compared to control; $P=0.0015$ ). Patients also reported statistically significant increases in "on" time without troublesome dyskinesia (+4.11 hours from baseline, +1.86 hours compared to control; $P=0.0059$ ) as well as "on" time without dyskinesia 
$(+3.37$ hours from baseline, +2.28 hours compared to control; $P=0.0142$ ). The average daily dose of DUOPA was 1117 $\mathrm{mg} /$ day compared with $1351 \mathrm{mg} /$ day of immediate-release oral CD-LD, meaning that the results were not likely to be attributable to significant increases in CD-LD dosing. 23

The long-term efficacy of DUOPA has also been observed in longer-term prospective and double-blinded trials. Fernandez et. al. completed a 54-week, open-label prospective study of 354 patients on DUOPA monotherapy, which revealed a significant decrease in mean daily "off" time and an increase in "on" time with and without troublesome dyskinesia. ${ }^{24} \mathrm{Of}$ the 272 patients who completed the trial from Weeks 4-54, "off" time decreased by $65 \%(-4.4 \pm 2.9$ hours per day hours; $P$ < 0.001), "on" time without troublesome dyskinesia increased by $62.9 \%(+4.8$ \pm 3.4 hours; $P<0.001)$, and "off" time decreased by $22.5 \%(-0.4 \pm 2.8$ hours per day; $(P=0.023)$. Slevin, et al. documented that, compared with immediate-release CD-LD in a 12-week double-blind, double-dummy trial, LCIG-naïve patients showed increased "on" time without troublesome dyskinesia (+2.19 hours/day; $\mathrm{P}<0.05)$ and decreased "off" time $(-2.27$ hours/day; $P<0.05) .25$ Furthermore, patients continuing on LCIG presented with sustained improvements in "off" time, further increases in "on" time, and quality-of-life metrics in long-term care.

DUOPA has been approved for use in 43 countries worldwide, which has provided opportunities for long-term patient experience studies. ${ }^{23}$ Monitoring a cohort of 59 PD patients on LCIG therapy for 7 years, Zibetti, et. al. found statistically significant improvements in UPDRS-related complications of therapy among the 41 patients who completed the study. ${ }^{26}$ Other significant findings included a decrease in the duration $130 \%$; $P=0.002)$, disability (48\%; $P=0.001)$, and pain $(78 \% ; P=0.020)$ from dyskinesia as well as a $49 \%$ decrease ( $P=0.001)$ in offperiod duration). $54 \%$ of patients reported an improvement in gait disorders, while only $18 \%$ reported improvement in dysarthria. Caceres-Redondo et. al. reported similar findings in a six-year observation of 29 LCIG patients, with significant improvements in UPDRS IV total score, "off" period duration, and dyskinesia. ${ }^{27}$
The most common adverse reactions were related to the placement of tubes for DUOPA administration and included implant site erosion, ulcer, infection, intestinal tube dislocation, occlusion/ kinking, PEG internal retention failure, intestinal complications (ischemia, hemorrhage, obstruction, perforation, ileus, peritonitis, pneumoperitoneum, and bezoar).22,26 Slevin et. al. reported an overall decrease in the incidence of adverse events throughout treatment. ${ }^{25}$ Other less-reported complications include the onset of "sleep attacks" during daily activities, an increase in the frequency of orthostatic hypotension (exacerbated with concurrent administration of antihypertensive drugs), as well psychiatric manifestations (hallucinations, psychoses, depression $-11 \%$ versus $3 \%$, and loss of impulse control). The treatment is also associated with an increased blood urea nitrogen (13\% in DUOPA versus $4 \%$ in oral intermediate-release LC) as well as increased creatinine phosphokinase $(17 \%$ versus $7 \%)$ of uncertain clinical significance. Non-selective MAO inhibitors are contraindicated due to a risk of hypertension and must be discontinued two weeks before treatment initiation. ${ }^{22}$

An emerging side effect that requires further study is a sub-acute to chronic generalized polyneuropathy linked to DUOPA therapy. $5 \%$ of patients (19/412) in DUOPA clinical trials developed a sensorimotor axonal polyneuropathy. There is emerging evidence that this phenomenon may be linked to impaired Vitamin B12 metabolism from long-term CD-LD therapy and not confined to LCIG. Ceravalo et. al. demonstrated that PD patients on long-term levodopa therapy were 2.38 times more likely to develop neuropathy compared to those with short-term LC therapy and no levodopa exposure, while having significantly higher doses of levodopa, higher levels of homocysteine, and lower levels of vitamin B12. ${ }^{28}$ These observations suggest careful examination, laboratory analysis, and potentially electromyographic studies prior to the initiation of any long-term levodopa therapy.

Pederson et al. established practical guidelines for instituting LCIG and evaluating, treating, and supporting LCIG patients in a Movement Disorders Clinic. ${ }^{29}$ Prior to discontinuation of their normal PD medications to begin the DUOPA trial period, staff should be aware of the anxiety that many patients will feel after stopping their medication. Prior to PEG-J placement, the physician and patient must work together to assess responsiveness to the therapy, willingness to undergo long-term therapy, and establish expectations about the efficacy of the treatment. Before discharge, staff should work to familiarize patients with all aspects of the device, stressing that the pump is pre-programmed with an individually titrated dose. The device should be checked biannually for proper functioning in addition to the patient's conference with their physician regarding the efficacy of the treatment. Patients should also be educated and provided with accouterments (i.e. cooling bags) that will allow them to continue LCIG therapy while maintaining their lifestyle and interests. Since the most frequent complications of the intervention involve maintenance of the tube itself, it is recommend that Movement Disorder centers coordinate with proceduralists (Gastroenterology/Interventional Radiology) to establish an emergency response center. ${ }^{29}$

\section{CONCLUSION}

Overall, RYTARY and intestinal DUOPA are effective therapies that serve to meet major unmet needs in the treatment of PD. There is extensive trial data showing RYTARY is safe and effective for advanced PD. Initial data on its use as a first-line treatment in early PD is promising, though further studies are needed comparing RYTARY to immediate release CD-LD in this patient population.

DUOPA is an effective and reversible alternative to DBS in patients with advanced PD. DUOPA has been used internationally for some time, and with recent FDA approval, it is gaining momentum in the United States. Its primary indication is to treat advanced PD patients who cannot control symptoms adequately with medical therapies available and who either are not candidates for DBS or who are unable or unwilling to go through the rigors of brain surgery. Further study and clinical experience in the U.S. will hopefully provide further data on its efficacy and safety profile. 


\section{REFERENCES}

1. Olanow, C. W., Stern, M. B., \& Sethi, K. (2009) The scientific and clinical basis for the treatment of parkinson disease (2009). Neurology, 72(21 Suppl 4), S1-136. doi:10.1212/ WNL.0b013e3181a1d44c [doi]

2. Jankovic, J., \& Stacy, M. (2007). Medical management of levodopa-associated motor complications in patients with parkinson's disease. CNS Drugs, 21(8), 677-692. doi:2185 [pii]

3. Deleu, D., Jacques, M., Michotte, Y., \& Ebinger, G. (1989). Controlled-release carbidopa/levodopa (CR) in parkinsonian patients with response fluctuations on standard levodopa treatment: Clinical and pharmacokinetic observations. Neurology, 39(11 Suppl 2), 88-92; discussion 95.

4. Reichmann, H., \& Emre, M. (2012). Optimizing levodopa therapy to treat wearing-off symptoms in parkinson's disease: Focus on levodopa/carbidopa/entacapone. Expert Review of Neurotherapeutics, 12(2), 119-131. doi:10.1586/ern.11.203 [doi]

5. Stocchi, F., Rascol, O., Kieburtz, K., Poewe, W., Jankovic, J., Tolosa, E., ... Olanow, C. W. (2010). Initiating levodopa/carbidopa therapy with and without entacapone in early parkinson disease: The STRIDE-PD study. Annals of Neurology, 68(1), 18-27. doi:10.1002/ana.22060 [doi]

6. Pahwa, R., Factor, S. A., Lyons, K. E., Ondo, W G., Gronseth, G., Bronte-Stewart, H., Quality Standards Subcommittee of the American Academy of Neurology. (2006). Practice parameter: Treatment of parkinson disease with motor fluctuations and dyskinesia (an evidence-based review): Report of the quality standards subcommittee of the american academy of neurology. Neurology, 66(7), 983-995. doi:66/7/983 [pii]

7. Stocchi, F., Vacca, L., Ruggieri, S., \& Olanow, C. W. (2005). Intermittent vs continuous levodopa administration in patients with advanced parkinson disease: A clinical and pharmacokinetic study. Archives of Neurology, 62(6), 905-910. doi:62/6/905 [pii]
8. Antonini, A., Isaias, I. U., Rodolfi, G., Landi, A., Natuzzi, F., Siri, C., \& Pezzoli, G. (2011). A 5-year prospective assessment of advanced parkinson disease patients treated with subcutaneous apomorphine infusion or deep brain stimulation. Journal of Neurology, 258(4), 579-585. doi:10.1007/s00415-0105793-z [doi]

9. Defer, G. L., Widner, H., Marie, R. M. Remy, P., \& Levivier, M. (1999). Core assessment program for surgical interventional therapies in parkinson's disease (CAPSIT-PD). Movement Disorders Official Journal of the Movement Disorder Society, 14(4), 572-584. doi:10.1002/15318257(199907)14:4<572::AIDMDS1005>3.0.CO;2-C

10. Lang, A. E., \& Widner, H. (2002). Deep brain stimulation for parkinson's disease: Patient selection and evaluation. Movement Disorders: Official Journal of the Movement Disorder Society, 17 Suppl 3, S94-101. doi:10.1002/mds.10149 [pii]

11. Hauser, R. A. (2012). IPX066: A novel carbidopa-levodopa extended-release formulation. Expert Review of Neurotherapeutics, 12(2), 133-140. doi:10.1586/ern.11.195 [doi]

12. Mao, Z., Hsu, A., Gupta, S., \& Modi, N. B. (2013). Population pharmacodynamics of IPX066: An oral extended-release capsule formulation of carbidopa-levodopa, and immediate-release carbidopa-levodopa in patients with advanced parkinson's disease. Journal of Clinical Pharmacology, 53(5), 523-531. doi:10.1002/jcph.63 [doi]

13. Pahwa, R., Lyons, K. E., Hauser, R. A., Fahn, S., Jankovic, J., Pourcher, E., ... APEX-PD Investigators. (2014). Randomized trial of IPX066, carbidopa/levodopa extended release, in early parkinson's disease. Parkinsonism \& Related Disorders, 20(2), 142-148. doi:10.1016/j. parkreldis.2013.08.017 [doi]
14. Hauser, R. A., Ellenbogen, A. L., Metman, L. V., Hsu, A., O'Connell, M. J., Modi, N. B., Gupta, S. K. (2011). Crossover comparison of IPX066 and a standard levodopa formulation in advanced parkinson's disease. Movement Disorders: Official Journal of the Movement Disorder Society, 26(12), 2246-2252. doi:10.1002/mds.23861 [doi]

15. Hauser, R. A., Hsu, A., Kell, S., Espay, A. J., Sethi, K., Stacy, M., PX066 ADVANCE-PD investigators. (2013). Extended-release carbidopa-levodopa (IPX066) compared with immediate-release carbidopa-levodopa in patients with parkinson's disease and motor fluctuations: A phase 3 randomised, double-blind trial. The Lancet. Neurology, 12(4), 346-356. doi:10.1016/S14744422(13)70025-5 [doi]

16. Stocchi, F., Hsu, A., Khanna, S., Ellenbogen, A., Mahler, A., Liang, G., ... Gupta, S. (2014). Comparison of IPX066 with carbidopalevodopa plus entacapone in advanced PD patients. Parkinsonism \& Related Disorders, 20(12), 1335-1340. doi:10.1016/j. parkreldis.2014.08.004 [doi]

17. Kestenbaum, M., \& Fahn, S. (2015). Safety of IPX066, an extended release carbidopalevodopa formulation, for the treatment of parkinson's disease. Expert Opinion on Drug Safety, 14(5), 761-767. doi:10.1517/14740338.2 015.1015986 [doi]

18. Waters, C. H., Nausieda, P., Dzyak, L., Spiegel, J., Rudzinska, M., Silver, D. E., ... Gupta, S. (2015). Long-term treatment with extendedrelease carbidopa-levodopa (IPX066) in early and advanced parkinson's disease: A 9-month open-label extension trial. CNS Drugs, 29(4), 341-350. doi:10.1007/s40263-015-0242-2 [doi] 